\section{OPEN ACCESS}

Edited by:

Tatjana Rundek,

University of Miami, United States

Reviewed by:

Dana Leifer,

Cornell University, United States Aristeidis H. Katsanos,

McMaster University, Canada

${ }^{*}$ Correspondence:

Alejandro Bustamante alejandro.bustamante@vhir.org

Specialty section

This article was submitted to

Stroke,

a section of the journa

Frontiers in Neurology

Received: 16 May 2019 Accepted: 04 November 2019 Published: 29 November 2019

Citation:

Palà E, Bustamante $A$,

Clúa-Espuny JL, Acosta J, Gonzalez-Loyola F, Ballesta-Ors J, Gill N, Caballero A, Pagola J,

Pedrote A, Muñoz MA and Montaner J (2019) N-Terminal Pro

B-Type Natriuretic Peptide's

Usefulness for Paroxysmal Atrial

Fibrillation Detection Among Populations Carrying Cardiovascular Risk Factors. Front. Neurol. 10:1226. doi: 10.3389/fneur.2019.01226

\title{
N-Terminal Pro B-Type Natriuretic Peptide's Usefulness for Paroxysmal Atrial Fibrillation Detection Among Populations Carrying Cardiovascular Risk Factors
}

\begin{abstract}
Elena Palà ${ }^{1}$, Alejandro Bustamante ${ }^{1,2 *}$, Josep Lluis Clúa-Espuny ${ }^{3,4}$, Juan Acosta ${ }^{5}$, Felipe Gonzalez-Loyola ${ }^{4,6}$, Juan Ballesta-Ors ${ }^{3,4}$, Natalia Gill ${ }^{1}$, Andrea Caballero ${ }^{7}$, Jorge Pagola ${ }^{2}$, Alonso Pedrote ${ }^{5}$, Miguel Angel Muñoz ${ }^{4,6}$ and Joan Montaner ${ }^{1}$
\end{abstract}

${ }^{1}$ Neurovascular Research Laboratory, Vall d'Hebron Institute of Research (VHIR)-Universitat Autónoma de Barcelona, Barcelona, Spain, ${ }^{2}$ Department of Neurology, Hospital Universitari Vall d'Hebron, Barcelona, Spain, ${ }^{3}$ Equip d'Atenció Primària Tortosa Est, SAP Terres de l'Ebre, Institut Català de la Salut, Tortosa, Spain, ${ }^{4}$ Unitat de Suport a la Recerca de Barcelona, Institut d'Investigació en Atenció Primària IDIAP Jordi Gol, Barcelona, Spain, ${ }^{5}$ Department of Cardiology, Hospital Universitario Virgen del Rocio, Sevilla, Spain, ${ }^{6}$ Gerència Atenció Primària de Barcelona, Institut Català de la Salut, Barcelona, Spain, ${ }^{7}$ Biochemical Department, Hospital Universitari Vall d'Hebron, Barcelona, Spain

Background: Atrial fibrillation (AF) systematic screening studies have not shown a clear usefulness in stroke prevention, as AF might present as paroxysmal and asymptomatic. This study aims to determine the usefulness of some blood-biomarkers to identify paroxysmal atrial fibrillation in the context of a screening programme.

Methods: A total of 100 subjects aged 65-75 years with hypertension and diabetes were randomly selected. AF was assessed by conventional electrocardiogram (ECG) and 4 weeks monitoring with a wearable Holter device $\left(\right.$ Nuubo $\left.{ }^{T M}\right)$. N-terminal pro B-type natriuretic peptide (NT-proBNP), apolipoprotein CIII (ApoC-III), von Willebrand factor (VWF), ADAMTS13, urokinase plasminogen activator surface receptor (UPAR), and urokinase plasminogen activator (UPA) were determined in serum/plasma samples and the levels were compared depending on AF presence and mode of detection.

Results: The AF prevalence in the studied population was found to be $20 \%$. In seven subjects, AF was only detected after 1 month of Holter monitoring (hAF group). NT-proBNP levels were higher in subjects with AF compared with subjects with no AF $(p<0.0001)$, even when only taking into account the hAF group $(p=0.031)$. No significant differences were found in the other biomarkers. The NT-proBNP $>95 \mathrm{pg} / \mathrm{ml}$ cut-off showed high sensitivity and specificity to detect AF (95\%, 66.2\%) or hAF (85.72\%, $66.2 \%$ ) and was found to be an independent predictor of AF and hAF in a logistic regression analysis. NT-proBNP correlated with AF burden ( $r=0.597, p=0.024)$.

Conclusion: NT-proBNP was elevated in AF cases not identified by ECG; thus, it may be used as a screening biomarker in asymptomatic high-risk populations, with a promising cut-off point of $95 \mathrm{pg} / \mathrm{ml}$ that requires further validation.

Keywords: stroke, atrial fibrillation, biomarker, screening, NT-proBNP 


\section{INTRODUCTION}

Atrial fibrillation (AF) is one of the fastest-growing cardiovascular epidemics of the twenty-first century (1). This heart rhythm disorder multiplies by five the risk of ischemic stroke (2), and its prevalence in the population over 60 years has been quantified at $10.9 \%$ (3). Anticoagulation may reduce stroke risk in AF individuals by $\sim 60 \%$ (4), but unfortunately, given its paroxysmal and asymptomatic nature at earlier stages, $\mathrm{AF}$ is often undiagnosed and/or undertreated when stroke occurs. In a previous study, the absolute prevalence of undiagnosed $\mathrm{AF}$ in individuals over 60 years was 3.4\%, representing up to $31 \%$ of the overall AF prevalence in our area $(5,6)$. International collaborations $(7,8)$ recommend opportunistic screening for $\mathrm{AF}$ in patients $>65$ years as a strategy to reduce stroke and death, but systematic screening programmes have failed to show a clear benefit and those strategies are lacking in high-risk populations (9-11).

Blood biomarkers might represent an alternative tool for AF screening, because some of them might have elevated circulating levels after AF paroxysms, a kind of "biological memory" offering a temporal advantage over electrical detection of the paroxysm itself. N-terminal pro B-type natriuretic peptide (NT-proBNP) has been found to be elevated in cardioembolic (CE) stroke (12) and to be associated with AF (13-16). Von Willebrand factor (vWF) was found to be elevated in non-valvular AF (17) and, together with apolipoprotein CIII (ApoC-III), was found to be predictor of stroke CE etiology [unpublished results from the Stroke-Chip study (18)]. ADAMTS-13 is the key regulator of VWF, and it has been associated with AF recurrence after cardioversion (19). Urokinase plasminogen activator surface receptor (UPAR) has been associated with AF prevalence (20) while its ligand, urokinase plasminogen activator (uPA), has not been studied in AF patients.

This study aims to determine the usefulness of the aforementioned biomarkers to identify AF individuals in the context of a screening programme, especially in cases of paroxysmal AF that would not be detected using a regular 12lead ECG.

\section{MATERIALS AND METHODS}

\section{Patients and Protocol}

AFRICAT (Atrial Fibrillation Research in CATalonia; NCT03188484) is an observational, multicenter, populationbased study with the aim of developing a screening programme to detect new cases of AF in high-risk individuals in primary care centers. In Phase I of the project, 100 subjects aged 65-75 with hypertension and diabetes were selected from primary care registers (e-cap), which include the clinical records from most Catalonia inhabitants between January 2016 and December 2017. In two different health service areas, Servei d'Atenció Primària (SAP) Muntanya and SAP Terres de l'Ebre, patients fulfilling the inclusion criteria were identified in primary care centers from each catchment [Centre d'Atenció Primària (CAP) Sant Rafael and CAP Trinitat Vella in SAP Muntanya; Equip d'Atenció Primària (EAP) Tortosa Est and EAP Tortosa Oest in SAP Terres de l'Ebre]. Physicians from these primary care centers were informed about the study protocol, and patients were invited by them to participate in the study until 50 patients were recruited in each catchment. Participants were ambulatory patients in a basal situation, meaning they were contacted at home to arrange a visit in their primary care center specifically for the study. During the appointments, patients received a comprehensive assessment consisting of clinical characteristics (demographic factors, vascular risk factors, medications, comorbidities, and vitals), electrographic assessment and a blood sample collection. Comorbid conditions were defined using standard outpatient and inpatient ICD-10 codes by electronic data capture, including pharmacy records, laboratory data, and emergency room and hospitalization diagnoses across all primary care centers and hospitals.

Individuals with chronic inflammatory diseases, cancer or dementia were excluded. AF was assessed by conventional electrocardiogram (ECG). Moreover, patients were monitored for 4 weeks with a wearable Holter device $\left(\mathrm{Nuubo}^{\mathrm{TM}}\right.$ ) as described previously (21). The Holter device was composed of a sensor that captured the electrocardiographic signal by noninvasive textile electrodes and a recorder of the signal that stored the information for subsequent detailed analysis. Participants were instructed by local trained researchers to wear the Holter for $23 \mathrm{~h}$ and recharge it for $1 \mathrm{~h}$ daily. After 4 weeks, individuals brought the Holter to their primary care center, and data were collected. Holter records, anonymized and encrypted, were sent together with the ECG for blinded reading to the Rhythm Disorders Unit at the Cardiology Department of Hospital Virgen del Rocio in Seville, to verify AF episodes. AF was defined following AHA guidelines as irregular R-R intervals without a $\mathrm{P}$ wave signal, lasting for more than $60 \mathrm{~s}$ (22). Individuals with previous AF were not excluded at this step, but Holter monitoring was optional in this subgroup.

Patients were classified and compared in five groups defined by past medical history (PMH) for AF, ECG findings and Holter AF detection as follows: no AF, PMH-ECG-Holter+ [Holter-detected AF (hAF)], PMH-ECG+, PMH+ECG-, and $\mathrm{PMH}+\mathrm{ECG}+$. Specific comparisons were done between the hAF and no AF groups.

The AFRICAT study protocol was approved by the clinical research ethics committees of IDIAP Jordi Gol (P15/047) and Hospital Universitari Vall d'Hebron [PR (AG) 133-2015]. All participants signed informed consent before inclusion. The study protocol conformed to the ethical guidelines of the 1975 Declaration of Helsinki.

\section{Biomarker Measurement}

Blood was collected into EDTA and serum tubes at the time of inclusion. After centrifugation at $1,500 \mathrm{~g}$ and $4^{\circ} \mathrm{C}$ for $15 \mathrm{~min}$, plasma and serum aliquots were frozen at $-80^{\circ} \mathrm{C}$ until biomarker determination.

Plasma NT-proBNP levels were determined by automated immunoassay in a COBAS c8000 (Roche Diagnostics); serum ApoC-III, plasma uPAR and plasma uPA by ELISA (Abnova, R\&D Systems, and Cloud-Clone); and plasma vWF and ADAMTS13 by a magnetic Luminex ${ }^{\circledR}$ assay (R\&D Systems). 
All assays were performed blinded to clinical information and according to the manufacturer's instructions. All samples were tested in duplicate and inter-assay variation was determined by a commercial control (Human Serum, male AB, USA origin from clotted, SIGMA, ref number H16914; Human plasma K2 EDTA, Innovative Research, ref number IPLA-N) tested in duplicate in each plate. When inter-assay variation was $>20 \%$ biomarker levels were standardized using the Z-score.

\section{Statistical Analysis}

Statistical analysis was conducted with SPSS version 20. Graphs were elaborated with GraphPad Prism 6. Data are expressed as number (\%) for categorical variables and as mean $\pm \mathrm{SD}$ or median (interquartile range) for continuous variables, depending on the data distribution. For univariate analysis, the MannWhitney $U$-test or Student's $t$-test was used for continuous variables, and the $\chi^{2}$ test was used for categorical variables. ANOVA or the Kruskal-Wallis test was used to compare $>2$ variables depending on variable distribution. Receiver operator characteristic (ROC) curves were configured to calculate sensitivity, specificity, and cut-off values. The Spearman test was used for correlations. Comparisons were first performed between $\mathrm{AF}$ patients and no AF patients and second between hAF and no AF patients (excluding other subtypes of AF). Binary logistic regression analysis was performed including variables associated with $\mathrm{AF}$ or hAF in univariate analysis.

\section{RESULTS}

\section{Patient Characteristics and AF Detection}

Clinical characteristics of the cohort can be found in Table 1. Coronary heart disease $(p=0.008)$, heart failure $(p=0.007)$, valvular diseases $(p=0.024)$, and use of anticoagulants $(p<0.0001)$ were more common in AF individuals. Of the 9 anticoagulated individuals, only one was anticoagulated for reasons other than AF (ischemic cardiopathy and deep venous thrombosis). The CHA2DS2-VASc score was higher in AF individuals $(p=0.035)$. Coronary heart disease ischemia was more common in hAF individuals compared to no AF $(p=0.032)$. No cases of heart failure, valvular disease or anticoagulation were present in this subgroup.

Of the included 100 individuals, 96 were monitored with ECG Holter. All patients with negative ECG were monitored. Median monitoring time was $457 \mathrm{~h}$. Six cutaneous adverse events related to wearing the Holter were noted; these consisted of a rash that normally disappeared with topical steroids. However, in two subjects, these adverse events prevented the monitoring being continued.

Of the 100 selected subjects, AF was present in 20 (11 newly detected within the present study) classified as follows: $7 \mathrm{PMH}-$ ECG-Holter+ (hAF), 4 PMH-ECG+, 2 PMH+ECG-, and 7 $\mathrm{PMH}+\mathrm{ECG}+($ Figure 1).

In 14 individuals (11 newly detected and $3 \mathrm{PMH}+\mathrm{ECG}+$ were monitored), AF was detected during Holter monitoring and AF burden was calculated. AF burden was defined as minutes being in AF divided by the total minutes of readable records and is expressed as a percentage. The median AF burden was $10.35 \%(1.00-56.50)$.

\section{Biomarker Analysis}

NT-proBNP, ApoC-III, sUPAR, vWF, and ADAMTS13 inter- and intra-assay variation was acceptable (coefficient of variation $<20$ ); hence, all the samples were included in the analysis. uPA levels were standardized because the inter-assay variation was higher than $20 \%$, and 9 samples were eliminated from statistical analysis

TABLE 1 | Clinical characteristics of the cohort and comparison according to atrial fibrillation diagnosis.

\begin{tabular}{|c|c|c|c|c|c|c|}
\hline & All patients (100) & AF (20) & No AF (80) & hAF (7) & $P$-value ${ }^{a}$ & $P$-value ${ }^{b}$ \\
\hline Age & $70(68-73)$ & $69(66-71.5)$ & 70 (68-73) & $70(65-72)$ & 0.273 & 0.655 \\
\hline Sex (\% female) & $33(33)$ & 7 (35) & $26(32.5)$ & $3(42.9)$ & 0.832 & 0.682 \\
\hline Tobacco & $20(20)$ & $4(20)$ & $16(20.3)$ & $1(14.3)$ & 1.000 & 1.000 \\
\hline Alcohol & $11(11)$ & $1(5)$ & $10(12.5)$ & $0(0)$ & 0.456 & 1.000 \\
\hline Dyslipidaemia & $81(81)$ & $16(80)$ & 65 (81.3) & $5(71.4)$ & 1.000 & 0.619 \\
\hline Coronary heart disease & $18(18)$ & $8(40)$ & $10(12.5)$ & 3 (42.9) & $0.008^{*}$ & $0.032^{\star}$ \\
\hline Heart failure & $3(3)$ & $3(15)$ & $0(0)$ & $0(0)$ & $0.007^{\star}$ & 1.000 \\
\hline Valvular disease & $4(4)$ & $3(15)$ & $1(1.3)$ & $0(0)$ & $0.024^{\star}$ & 0.767 \\
\hline Previous stroke & $6(6)$ & $2(10)$ & $4(5)$ & $0(0)$ & 0.597 & 1.000 \\
\hline Anticoagulation & $9(9)$ & $8(40)$ & $1(1.3)$ & $0(0)$ & $0.000^{\star}$ & 0.920 \\
\hline Antiplatelets & $50(50)$ & $7(35)$ & $43(53.8)$ & $3(42.9)$ & 0.134 & 0.702 \\
\hline Familiar history FA & $5(5)$ & $2(10)$ & $3(3.9)$ & $0(0)$ & 0.273 & 1.000 \\
\hline SBP, mm Hg & 143.50 (134-153) & 140.5 (127.5-162.5) & $144(134-151.75)$ & 140 (124-168) & 0.973 & 0.773 \\
\hline DBP, mm Hg & $78.95 \pm 10.09$ & $80.95 \pm 10.85$ & $78.45 \pm 9.90$ & $79.14 \pm 6.66$ & 0.332 & 0.857 \\
\hline Heart rate & $77.94 \pm 15.70$ & $77.25 \pm 16.94$ & $78.11 \pm 15.48$ & $73 \pm 22.14$ & 0.724 & 0.421 \\
\hline $\mathrm{CHA}_{2} \mathrm{DS}_{2}$-VASc & $4(3-4)$ & $4(3-4)$ & $4(3-5)$ & $4(3-5)$ & $0.035^{\star}$ & 0.284 \\
\hline
\end{tabular}

a $P$-value comparison AF vs. no AF.

${ }^{b} P$-value comparison hAF vs no $A F$.

${ }^{*} P<0.05$. AF, atrial fibrillation; DBP, diastolic blood pressure; $h A F$, Holter-detected atrial fibrillation; SBP, systolic blood pressure. 


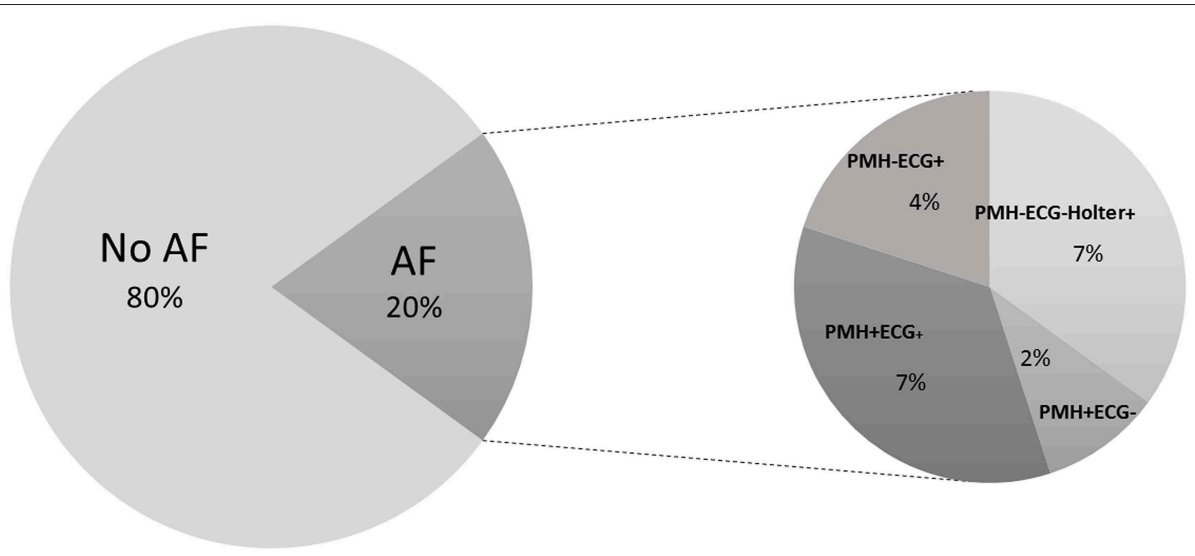

FIGURE 1 | Subject classification. Individuals were classified depending on AF presence and subgroups were made depending on AF detection with different methods and previous medical history.

TABLE 2 | Biomarker levels and comparisons between different groups.

\begin{tabular}{|c|c|c|c|c|c|}
\hline & AF (20) & No AF (80) & hAF (7) & $P$-value ${ }^{a}$ & $P$-value ${ }^{b}$ \\
\hline NT-proBNP (pg/ml) & $\begin{array}{c}643.65 \\
(155.72-1339.25)\end{array}$ & 64.28 (37.08-133.10) & $\begin{array}{c}128.30 \\
(97.69-191.8)\end{array}$ & $<0.0001^{\star}$ & $0.03^{*}$ \\
\hline ApoC-III (ng/ml) & $\begin{array}{c}107,594 \\
(92,501-129262.06)\end{array}$ & $104,450(766,64-130,393)$ & $\begin{array}{c}105,352 \\
(91,956-132,268)\end{array}$ & 0.496 & 0.574 \\
\hline ADAMTS13 (ng/ml) & $1131.24 \pm 471.419$ & $1221.41 \pm 459.80$ & $1271.68 \pm 561.01$ & 0.437 & 0.786 \\
\hline VWF (ng/ml) & $0.139 \pm 0.052$ & $0.139 \pm 0.706$ & $0.146 \pm 0.060$ & 0.987 & 0.802 \\
\hline uPA $(s t d)^{c}$ & $2.01(1.41-2.39)$ & $1.60(1.38-2.35)$ & 1.68 (1.29-2.35) & 0.720 & 0.868 \\
\hline uPAR (pg/ml) & $\begin{array}{c}2220.80 \\
(557.74-2810.52)\end{array}$ & 2267.54 (1518.33-2763.05) & $\begin{array}{c}2246.12 \\
(1926.03-259.23)\end{array}$ & 0.689 & 0.918 \\
\hline
\end{tabular}

a P-value comparison AF vs. no $A F$.

${ }^{b} P$-value comparison hAF vs. no AF.

${ }^{c}$ Ninety-one individuals included in the UPA analysis (73 no AF and 18 AF, of whom 6 were hAF).

${ }^{*} P<0.05$. AF, atrial fibrillation; ApoC-III, Apolipoprotein C-III; hAF, Holter-detected group; NT-proBNP, N-terminal pro B-type natriuretic peptide; vWF, von Willebrand factor; uPA, urokinase plasminogen activator; UPAR, urokinase plasminogen activator surface receptor.

because their duplicates showed $\mathrm{CV}>20 \%$. Median levels and comparisons are shown in Table 2.

\section{AF vs. No AF}

NT-proBNP was significantly higher in individuals with AF compared with no AF $[643.65 \mathrm{pg} / \mathrm{ml}$ (IQR 155.72-1339.25) vs. $64.28 \mathrm{pg} / \mathrm{ml}$ (IQR 37.08-133.10), $p<0.0001$; Figure 2A], while the remaining biomarkers were not different between the two groups (Supplementary Figures 1, 2). The NT-proBNP distribution across the different subgroups is shown in Figure 2B. In addition, AF burden was correlated with NT-proBNP $(r=$ $0.597, p=0.024$; Figure 2D). The discriminating ability (area under the ROC curve) of NT-proBNP was 0.900 (95\% CI, 0.82740.9726, $p<0.0001$ ) to detect any AF (Figure 3A). The cut-off point of NT-proBNP of $>95 \mathrm{pg} / \mathrm{ml}$ showed $95 \%$ sensitivity, $66.2 \%$ specificity, $41.3 \%$ positive predictive value (PPV), and $98.1 \%$ negative predictive value (NPV) to detect any AF (Figure 3C).

Anticoagulants $(\mathrm{OR}=18.90 ; 95 \% \mathrm{CI}, 2.10-169.83 ; p=0.09)$ and NT-proBNP > 95 pg/ml $(\mathrm{OR}=22.42 ; 95 \% \mathrm{CI}, 2.74-183.15 ; p$ $=0.04$ ) were the only independent predictors of AF in the logistic regression analysis performed in the whole cohort. CHA2DS2VASc was an independent AF predictor when considered alone in the regression analysis $(\mathrm{OR}=2.29 ; 95 \% \mathrm{CI} 1.16-4.44 ; p=$ 0.017 ). However, after the addition of NT-proBNP $>95 \mathrm{pg} / \mathrm{ml}$, CHA2DS2-VASc was not significant in the model.

Alternatively, the cut-off point of NT-proBNP of $>125 \mathrm{pg} / \mathrm{ml}$, which is recommended for heart failure diagnosis in the nonacute setting, showed $85 \%$ sensitivity and $73.8 \%$ specificity, $44.7 \%$ PPV and 95.2\% NPV. NT-proBNP $>125 \mathrm{pg} / \mathrm{ml}$ was also an independent predictor of $\mathrm{AF}(\mathrm{OR}=8.85 ; 95 \% \mathrm{CI}, 2.17-35.95$; $p=0.017$ ).

Sensitivity analysis was performed while excluding the patient who was anticoagulated for reasons other than $\mathrm{AF}$, and the results were similar.

\section{hAF vs. No AF}

NT-proBNP levels were different between no AF and hAF [64.28 $\mathrm{pg} / \mathrm{ml}$ (IQR 37.08-133.10) vs. 128.3 pg/ml (IQR 97.69-191.3), $p=0.031$; Figure 2C] while other biomarkers did not show differences (Supplementary Figures 1, 2). Patients without AF 


\section{A}

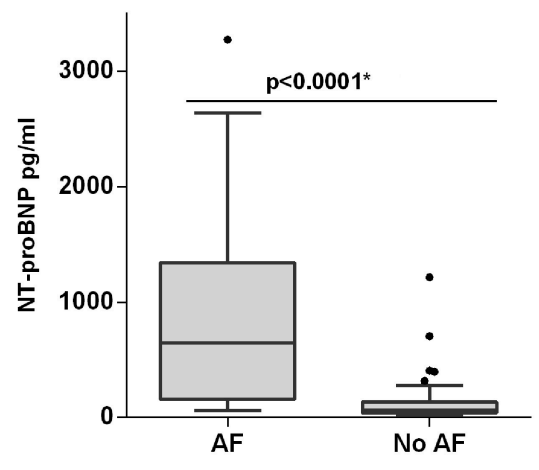

C

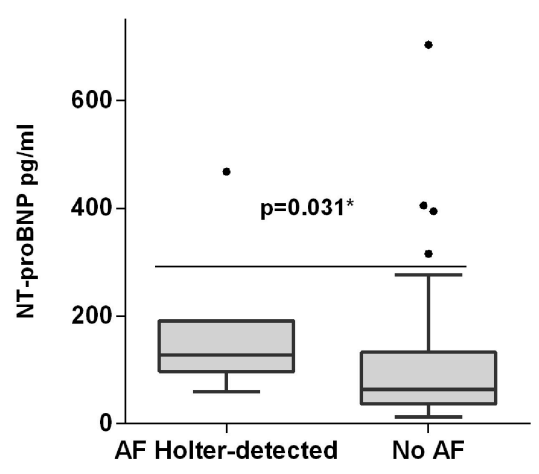

B

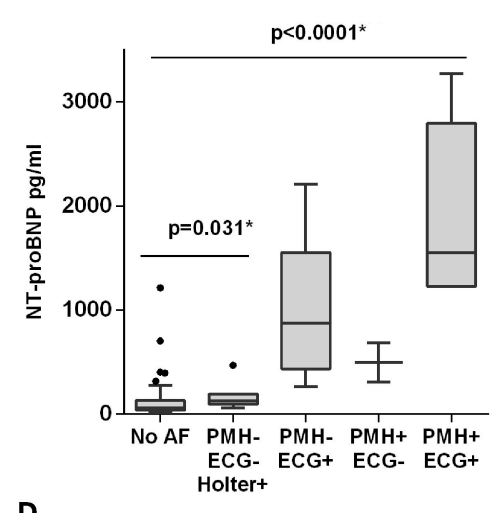

D

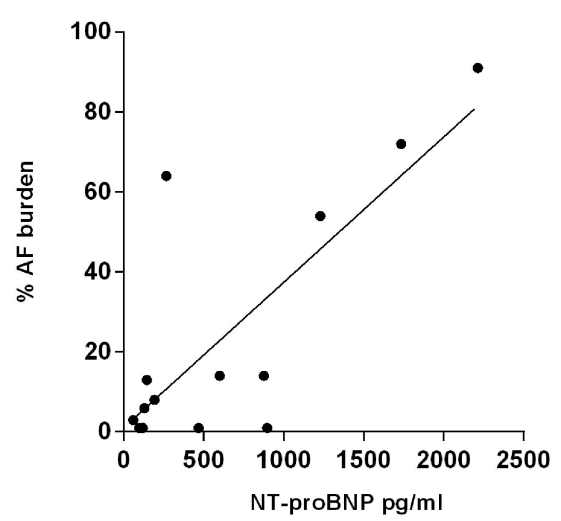

FIGURE 2 | Plasma NT-proBNP level distribution. (A) Boxplot distribution between AF and no AF. (B) Boxplot distribution between different groups depending on past medical history $(\mathrm{PMH})$ for AF, ECG findings, and Holter monitoring. (C) Boxplot distribution between Holter-detected AF (hAF) and no AF. (D) Correlation between NT-proBNP levels and AF burden. ${ }^{\star} P<0.05$.

and with a Holter monitoring time below the median $(457 \mathrm{~h})$ were removed and all aforementioned comparisons were tested again, with similar results (data not shown). The discriminating ability (area under the ROC curve) of NT-proBNP was 0.7464 (95\% CI 0.6087-0.8841, $p=0.031$ ) to detect hAF (Figure 3B). The cut-off point of NT-proBNP of $>95 \mathrm{pg} / \mathrm{ml}$ showed 85.7 $\%$ sensitivity, $66.2 \%$ specificity, $18.2 \% \mathrm{PPV}$, and $98.1 \% \mathrm{NPV}$ to detect hAF (Figure 3C).

The logistic regression analysis performed on this subgroup confirmed that NT-proBNP $\geq 95 \mathrm{pg} / \mathrm{ml}$ was an independent predictor of Holter-detected atrial fibrillation (OR, 11.778; 95\% CI, $1.35-102.85 ; p=0.026$ ). No clinical variable was an independent predictor of AF detection.

Alternatively, the cut-off point of NT-proBNP of $>125$ $\mathrm{pg} / \mathrm{ml}$ showed $55.1 \%$ sensitivity, $73.8 \%$ specificity, $16 \% \mathrm{PPV}$, and 95.2\% NPV. NT-proBNP $>125 \mathrm{pg} / \mathrm{ml}$ also was an independent predictor of $\mathrm{AF}(\mathrm{OR}=8.85 ; 95 \% \mathrm{CI} 2.17-35.95 ; p=0.017)$. NTproBNP $>125 \mathrm{pg} / \mathrm{ml}$ was not an independent predictor of hAF.

\section{DISCUSSION}

The prevalence of AF in our population was 20\%, of which $55 \%$ were newly detected AF individuals. This prevalence is higher than reported in previous screening studies (from 2.3 to $12.3 \%)(10,11)$. Most such studies have only used ECG or pulse devices instead of long-term monitoring, so they have missed cases of paroxysmal AF. Moreover, we used a targeted screening approach, focussing on a high-risk population with advanced age and other conditions related to AF, such as hypertension and diabetes, who should present a higher AF prevalence (23). Longer monitoring in a high-risk population might therefore be a good strategy to further explore in larger cohorts. Other studies using long-term monitoring with insertable cardiac devices or pacemakers in patients without prior history of AF have also shown a higher prevalence of AF (24). In fact, in the recent REVEAL AF trial, detection rates of AF rose with the monitoring time (25). Moreover, in patients with cryptogenic stroke, longterm monitoring was found to be associated with higher rates of AF detection, with a significant impact in secondary prevention (26). However, although it could become the gold standard for AF detection, long-term monitoring devices are expensive and unsuitable for application in primary care. In that sense, biomarkers might be used as cost-effective alternatives for $\mathrm{AF}$ systematic screening.

The present study suggests that NT-proBNP might be used as a screening biomarker to detect even paroxysmal AF in 
A

ROC curve: AF vs no AF

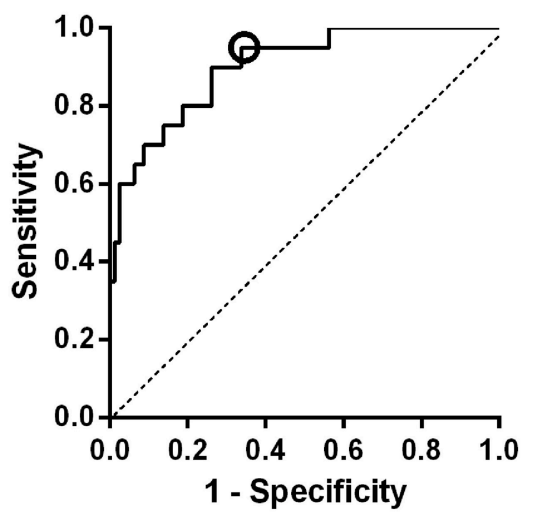

B

\section{ROC curve: hAF vs no AF}

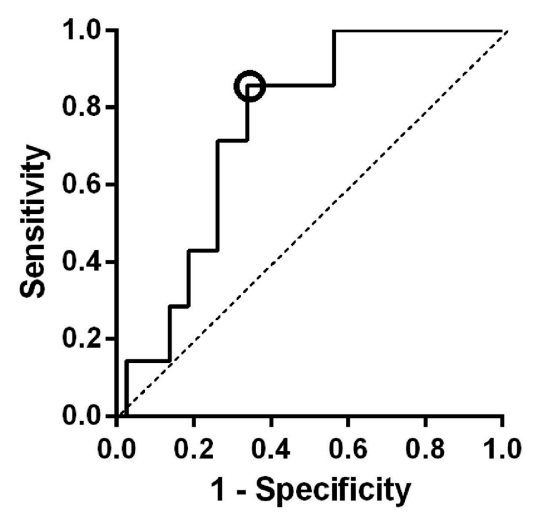

C

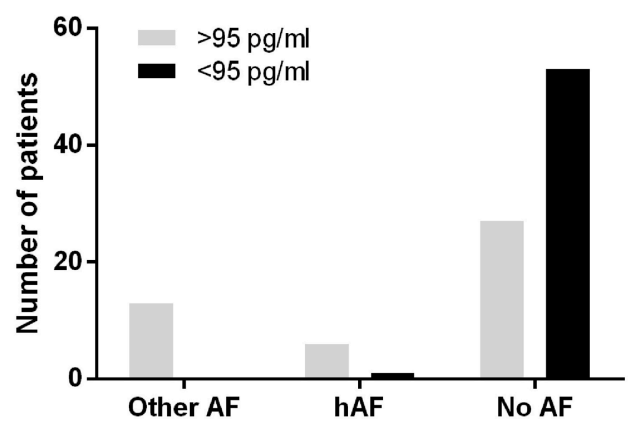

FIGURE 3 | NT-proBNP discrimination power. (A) ROC curve: ability to discriminate between AF and no AF. (B) ROC curve, ability to discriminate between Holter-detected AF and no AF. The cut-off with the best specificity and sensitivity to detect hAF is marked with a circle in the two curves (95 pg/ml), as it is the cut-off value that was used to calculate sensitivity, specificity and predictive values. (C) Discrimination power of the NT-proBNP cut-off value of 95 pg/ml. All AF individuals had NT-proBNP plasma levels below the previous cut-off (except for a patient with paroxysmal AF), and the majority of no AF patients had values above the cut-off.

asymptomatic, high-risk individuals. The focus of the present study was to assess the predictive ability of NT-proBNP and other biomarkers to diagnose paroxysmal atrial fibrillation and be used as the first step of a screening workflow. In fact, the best screening biomarker in this context would be able to detect those cases impossible to notice with other available tools, such opportunistic ECG or hand-held ECG devices. It should be mentioned that paroxysmal AF (pAF) patients also can have increased risk of stroke $(27,28)$. Moreover, pAF may progress to permanent AF within a year in almost $20 \%$ of cases in the aging population $(29,30)$.

Previous studies have suggested NT-proBNP as a screening tool (14-16), but a limited length of monitoring might have misclassified some cases of paroxysmal atrial fibrillation. Our study overcame this limitation by recording patient's heart rhythm continuously for 30 days using a Holter monitor, allowing us to detect more AF cases. This is to our knowledge the longest period over which asymptomatic individuals have been monitored to detect atrial fibrillation in a biomarker study, if we exclude studies using pacemakers (13). The limitation of pacemakers is that their use is restricted to very specific populations with cardiac diseases that could affect some biomarker levels.

In the pilot study of Seegers et al. (14), in addition to a limited 7-day Holter ECG for the evaluation of paroxysmal AF, patients were preselected as those with lowest and highest NTproBNP quartiles, overestimating the discriminatory value of the biomarker. In our study, inclusion was performed before any biomarker determination, and 4-week Holter monitoring was used as the gold standard to assess the usefulness of blood-based biomarkers in AF screening. Although individuals with AF detected by other methods were not excluded from the whole study, to increase the statistical power, specific comparisons were performed while excluding these patients and only taking into account AF cases detected with long-term monitoring, i.e., cases with neither previous AF history nor detection during electrocardiographic assessment. Even though few patients were part of this group, specific comparisons showed the usefulness of NT-proBNP to identify those patients outside AF paroxysms. After validating those results with a larger cohort, NT-proBNP could be implemented as part 
of a screening programme to indicate long-term monitoring in patients with a risk biological profile. Such a protocol would identify patients at risk of AF who would benefit from anticoagulation, thereby improving primary stroke prevention by preventing cardioembolic stroke, which is associated with the poorest disability and mortality rates among stroke etiologies (31).

Additionally, we proposed for the first time a specific cut-off point for atrial fibrillation screening. Seegers et al. (14) suggested that NT-proBNP could be used for stroke prevention after finding increased values in AF individuals, without indicating any cutoff. Svenberg et al. (15) and Ghazal et al. (16) proposed a cut-off point of NT-proBNP $>125 \mathrm{pg} / \mathrm{ml}$, which is the recommended for heart failure in the non-acute setting (32). Here, NT-proBNP >95 $\mathrm{pg} / \mathrm{ml}$ reached high sensitivity (95\%) even to detect paroxysmal AF individuals (85.7\%). In fact, in our cohort, this cut-off point only misclassified one paroxysmal AF individual. Although the NT-proBNP biomarker is not specific for AF and might be elevated in other cardiac diseases and renal dysfunction (33), a high sensitivity is more useful than specificity for screening purposes. NT-proBNP might be rapid and easy to use to identify all patients who need follow-up Holter monitoring since it is already widely employed as a point-of-care analysis tool for heart failure.

Although it might be suspected that elevated NT-proBNP levels were due to a higher burden of heart failure and other cardiovascular diseases in the atrial fibrillation subjects of our cohort, in the hAF group, there were few individuals with known comorbidities that could affect NT-proBNP levels. In fact, in this group, there were no cases of heart failure, and there were only differences in coronary heart disease when compared to no AF individuals. In addition, the study was carried out at the outpatient clinic, and therefore, no patient was in a situation of acute decompensated heart failure. This, combined with multivariate analysis, confirmed the independent value of NT-proBNP in diagnosing paroxysmal atrial fibrillation.

ApoC-III, vWF, ADAMTS13, uPAR, and uPA were not elevated in $\mathrm{AF}$ individuals in the present study. Although associated with cardioembolic stroke or AF recurrence, these biomarkers might not be useful in the context of AF screening. However, it would be interesting to perform further discovery studies to find new biomarkers that might be used in combination with NT-proBNP, increasing its specificity and sensitivity.

Interestingly, the present study showed that a higher level of NT-proBNP was correlated with higher AF burden, a conclusion based on continuous Holter monitoring. This tendency was also reflected in the distribution of NT-proBNP levels between the groups, with higher levels in individuals with a previous medical history of AF compared with individuals with hAF, as shown in Figure 1B. In fact, some studies have suggested that AF burden is related to higher stroke risk (34), and other biomarker-based models have confirmed NT-proBNP as a stroke risk surrogate (35).

Although the present study was performed in asymptomatic patients, NT-proBNP might also be useful for detecting cryptogenic stroke patients with occult AF. Recently, the negative results from clinical trials of embolic strokes of undetermined source (ESUS) patients receiving non-vitamin $\mathrm{K}$ antagonists (NOACs) $(36,37)$ indicate that this is a heterogeneous group of patients who need different management, only a minority of whom have undetected paroxysmal AF (38). In fact, the ongoing ARCADIA trial (39) will test the use of anticoagulants in ESUS patients with atrial cardiopathy using high NT-proBNP (>250 $\mathrm{pg} / \mathrm{ml}$ ) as one of the inclusion criteria.

Our study has some limitations. First, the participant selection was not randomized and might have suffered some selection bias: the high rates of $\mathrm{AF}$ might not be attributed only to the selection of high-risk patients and long-term monitoring but also to patients with more cardiac comorbidities being more prone to participate. The small sample size and the small number of patients with AF suggest that these results should be interpreted as hypothesis-generating. The protocol of the study did not include an echocardiography, missing the opportunity to establish correlations between biomarkers and left atrial diameter. Finally, the included population presented specific risk factors, and the results may not be directly applicable to populations with other combinations of risk factors. Further validation studies in larger cohorts are needed.

\section{CONCLUSIONS}

NT-proBNP was found to be elevated in AF individuals compared to controls. This biomarker also detected cases of AF not previously known or detected by ECG. NT-proBNP may be useful as a screening biomarker in asymptomatic high-risk populations with a promising cut-off point of $95 \mathrm{pg} / \mathrm{ml}$ that requires further validation. A screening strategy based on NTproBNP, alone or in combination with other biomarkers, might be of interest for the early initiation of anticoagulants, which could reduce cardioembolic stroke consequences.

\section{DATA AVAILABILITY STATEMENT}

The raw data supporting the conclusions of this manuscript will be made available by the authors, without undue reservation, to any qualified researcher.

\section{ETHICS STATEMENT}

The studies involving human participants were reviewed and approved by Ethics Committee of Research Institute IDIAP Jordi Gol (P15/047/2015) and Hospital Universitari Vall d'Hebron Clinical Research Ethics Committee [PR(AG)133-2015]. The patients/participants provided their written informed consent to participate in this study.

\section{AUTHOR CONTRIBUTIONS}

$\mathrm{AB}$, JC-E, FG-L, JB-O, and MM recruited patients. JA and AP read Holter-ECG to verify AF episodes. EP, NG, and AC performed biomarker measurements. AB, JC-E, JP, MM, and JM planned the whole project and drafted the study protocol. EP 
performed statistical analysis and drafted the manuscript. All authors have critically reviewed the manuscript and approved the final article version.

\section{FUNDING}

The study received a research grant by Fundació Marató de TV3 in the research call La Marató 2014: malalties del cor. Grant number: 201528-30-31-3. AB was supported by a Juan Rodés

\section{REFERENCES}

1. Stevens E, Emmett E, Wang Y, McKevitt C, DA Wolfe C. The Burden of Stroke in Europe Report. King's College London for the Stroke Alliance for Europe (SAFE), Brussels (2017).

2. Wolf P, Dawber T, Thomas HJ, Kannel W. Epidemiologic assessment of chronic atrial fibrillation and risk of stroke: the Framingham study. Neurology. (1978) 28:973-7. doi: 10.1212/WNL.28.10.973

3. Roso-llorach A, Clua-espuny JL, Panisello-tafalla A, Lucas-noll J, Lo C, Gallofre M, et al. Prevalence of undiagnosed atrial fibrillation and of that not being treated with anticoagulant drugs : the AFABE study. Rev Esp Cardiol. (2013) 66:545-52. doi: 10.1016/j.rec.2013.03.003

4. Hart RG, Pearce LA, Aguilar M. Meta-analysis: antithrombotic therapy to prevent stroke in patients who have nonvalvular atrial fibrillation. Ann Intern Med. (2007) 146:857-67. doi: 10.7326/0003-4819-146-12-200706190-00007

5. Panisello-tafalla A, Clua-espuny JL, Gil-guillen VF, González-henares A, Queralt-tomas ML, López-pablo C, et al. Results from the registry of atrial fibrillation (AFABE): gap between undiagnosed and registered atrial fibrillation in adults - ineffectiveness of oral anticoagulation treatment with VKA. Biomed Res Int. (2015) 2015:134756. doi: 10.1155/2015/134756

6. Morillas P, Pallarés V, Llisterri L, Sanchis C, Sánchez T. Prevalence of atrial fibrillation and use of antithrombotics in hypertensive patients aged $\geq 65$ years. The FAPRES Trial. Rev Esp Cardiol. (2010) 63:943-50. doi: 10.1016/S1885-5857(10)70188-2

7. Kirchhof P, Benussi S, Kotecha D, Ahlsson A, Atar D, Casadei B, et al. 2016 ESC guidelines for the management of atrial fibrillation developed in collaboration with EACTS. Rev Esp Cardiol. (2017) 70:50. doi: 10.1016/j.rec.2016.11.033

8. Freedman B. Screening for atrial fibrillation: a report of the AFSCREEN international collaboration. Circulation. (2017) 135:1851-67. doi: 10.1161/CIRCULATIONAHA.116.026693

9. Moran PS, Teljeur C, Ryan M, Smith SM. Systematic screening for the detection of atrial fibrillation. Cochrane Database Syst Rev. (2016) 2016:CD009586. doi: 10.1002/14651858.CD009586.pub3

10. Svennberg E, Engdahl J, Al-Khalili F, Friberg L, Frykman V, Rosenqvist M. Mass screening for untreated atrial fibrillation the STROKESTOP study. Circulation. (2015) 131:2176-84. doi: 10.1161/CIRCULATIONAHA.114.014343

11. Lowres N, Neubeck L, Redfern J, Ben Freedman S. Screening to identify unknown atrial fibrillation: a systematic review. Thromb Haemost. (2013) 110:213-22. doi: 10.1160/TH13-02-0165

12. Llombart V, Antolin-Fontes A, Bustamante A, Giralt D, Rost NS, Furie K, et al. B-type natriuretic peptides help in cardioembolic stroke diagnosis. Stroke. (2015) 46:1187-95. doi: 10.1161/STROKEAHA.114.008311

13. Wakula P, Neumann B, Kienemund J, Thon-Gutschi E, Stojakovic T, Manninger M, et al. CHA2DS2-VASc score and blood biomarkers to identify patients with atrial high-rate episodes and paroxysmal atrial fibrillation. Europace. (2017) 19:544-51. doi: 10.1093/europace/euw101

14. Seegers J, Zabel M, Grüter T, Ammermann A, Weber-Krüger M, Edelmann F, et al. Natriuretic peptides for the detection of paroxysmal atrial fibrillation. Open Hear. (2015) 2:e000182. doi: 10.1136/openhrt-2014-000182

15. Svennberg E, Henriksson P, Engdahl J, Hijazi Z, Al-Khalili F, Friberg L, et al. $\mathrm{N}$-terminal pro B-type natriuretic peptide in systematic screening for atrial fibrillation. Heart. (2017) 103:1271-7. doi: 10.1136/heartjnl-2016-310236 research contract (JR16/00008) from Instituto de Salud Carlos III. EP has received a predoctoral grant from Vall D'Hebron Institute of Research.

\section{SUPPLEMENTARY MATERIAL}

The Supplementary Material for this article can be found online at: https://www.frontiersin.org/articles/10.3389/fneur. 2019.01226/full\#supplementary-material

16. Ghazal F, Theobald H, Rosenquist M, Al-Khalili F. Assessment of N terminal pro-brain natriuretic peptide level in the screening for atrial fibrillation in the primary health care. PLoS ONE. (2019) 14:e0212974. doi: 10.1371/journal.pone.0212974

17. Muñoz-Cortés G, Viveros-Sandoval ME, Areán-Martínez C, Vega-Gómez HE, López-Castañeda SE, et al. Von Willebrand factor plasma levels variability in nonvalvular atrial fibrillation. J Atr Fibrillation. (2014) 7:1124. doi: 10.4022/jafib.1124

18. Bustamante A, López-Cancio E, Pich S, Penalba A, Giralt D, GarcíaBerrocoso T, et al. Blood biomarkers for the early diagnosis of stroke: the stroke-chip study. Stroke. (2017) 48:2419-25. doi: 10.1161/STROKEAHA.117. 017076

19. Freynhofer MK, Bruno V, Jarai R, Gruber S, Höchtl T, Brozovic I, et al. Levels of von Willebrand factor and ADAMTS13 determine clinical outcome after cardioversion for atrial fibrillation. Thromb Haemost. (2011) 105:435-43. doi: 10.1160/TH10-09-0615

20. Ichihara N, Miyamura M, Maeda D, Fujisaka T, Fujita SI, Morita H, et al. Association between serum soluble urokinase-type plasminogen activator receptor and atrial fibrillation. J Arrhythmia. (2017) 33:469-74. doi: 10.1016/j.joa.2017.05.003

21. Pagola J, Juega J, Francisco-Pascual J, Moya A, Sanchis M, Bustamante A, et al. Yield of atrial fibrillation detection with Textile Wearable Holter from the acute phase of stroke: pilot study of Crypto-AF registry. Int J Cardiol. (2018) 251:45-50. doi: 10.1016/j.ijcard.2017.10.063

22. January CT, Wann LS, Alpert JS, Calkins H, Cigarroa JE, Cleveland JC Jr, et al. 2014 AHA/ACC/HRS guideline for the management of patients with atrial fibrillation: executive summary: a report of the American College of Cardiology/American Heart Association Task Force on practice guidelines and the Heart Rhythm Society. Circulation. (2014) 130:2071-04. doi: 10.1161/CIR.0000000000000040

23. Lau DH, Nattel S, Kalman JM, Sanders P. Modifiable risk factors and atrial fibrillation. Circulation. (2017) 136:583-96. doi: 10.1161/CIRCULATIONAHA.116.023163

24. Van Gelder IC, Healey JS, Crijns HJGM, Wang J, Hohnloser SH, Gold MR, et al. Duration of device-detected subclinical atrial fibrillation and occurrence of stroke in ASSERT. Eur Heart J. (2017) 38:1339-44. doi: 10.1093/eurheartj/ehx042

25. Reiffel JA, Verma A, Kowey PR, Halperin JL, Gersh BJ, Wachter R, et al. Incidence of previously undiagnosed atrial fibrillation using insertable cardiac monitors in a high-risk population: the REVEAL AF study. JAMA Cardiol. (2017) 2:1120-7. doi: 10.1001/jamacardio.2017.3180

26. Tsivgoulis G, Katsanos AH, Mac GB, Köhrmann M, Ricci BA, Tsioufis K, et al. Prolonged cardiac rhythm monitoring and secondary stroke prevention in patients with cryptogenic cerebral ischemia. Stroke. (2019) 50:2175-80. doi: 10.1161/STROKEAHA.119.025169

27. Friberg L, Hammar N, Rosenquist M. Stroke in paroxysmal atrial fibrillation: report from the Stockholm Cohort of Atrial Fibrillation. Eur Heart J. (2010) 31:967-75. doi: 10.1093/eurheartj/ehn599

28. Lilli A, Di Cori A, Zacà V. Thromboembolic risk and effect of oral anticoagulation according to atrial fibrillation patterns: a systematic review and meta-analysis. Clin Cardiol. (2017) 40:641-7. doi: 10.1002/clc. 22701

29. Proietti R, Hadjis A, Alturki A, Thanassoulis G, Roux JF, Verma A, et al. A systematic review on the progression of paroxysmal to persistent atrial 
fibrillation: Shedding new light on the effects of catheter ablation. JACC Clin Electrophysiol. (2015) 1:105-15. doi: 10.1016/j.jacep.2015.04.010

30. De Sisti A, Leclercq JF, Halimi F, Fiorello P, Bertrand C, Attuel P. Evaluation of time course and predicting factors of progression of paroxysmal or persistent atrial fibrillation to permanent atrial fibrillation. Pacing Clin Electrophysiol. (2014) 37:345-55. doi: 10.1111/pace.12264

31. Freeman WD, Aguilar MI. Prevention of cardioembolic stroke. Neurotherapeutics. (2011) 8:488-502. doi: 10.1007/s13311-011-0048-y

32. Ponikowski P, Voors AA, Anker SD, Bueno H, Cleland JGF, Coats AJS, et al. 2016 ESC Guidelines for the diagnosis and treatment of acute and chronic heart failure. Eur Heart J. (2016) 37:2129-200. doi: 10.1093/eurheartj/ehw128

33. Troughton RW, Lewis LK, Yandle TG, Pemberton CJ, Nicholls MG. B-type natriuretic peptides: Looking to the future. Ann Med. (2011) 43:188-97. doi: $10.3109 / 07853890.2010 .526630$

34. Chen LY, Chung MK, Allen LA, Ezekowitz M, Furie KL, McCabe P, et al. Atrial fibrillation burden: moving beyond atrial fibrillation as a binary entity: a scientific statement from the american heart association. Circulation. (2018) 137:e623-44. doi: 10.1161/CIR.0000000000000568

35. Hijazi Z, Oldgren J, Lindbäck J, Alexander JH, Connolly SJ, Eikelboom $\mathrm{JW}$, et al. The novel biomarker-based $\mathrm{ABC}$ (age, biomarkers, clinical history)-bleeding risk score for patients with atrial fibrillation: a derivation and validation study. Lancet. (2016) 387:2302-11. doi: 10.1016/S0140-6736(16)00741-8

36. Hart RG, Sharma M, Mundl H, Kasner SE, Bangdiwala SI, Berkowitz SD, et al. Rivaroxaban for stroke prevention after embolic stroke of undetermined source. N Engl J Med. (2018) 378:2191-201. doi: 10.1056/NEJMoa1802686
37. Hart RG, Catanese L, Perera KS, Ntaios G, Connolly SJ. Embolic stroke of undetermined source: a systematic review and clinical update. Stroke. (2017) 48:867-72. doi: 10.1161/STROKEAHA.116. 016414

38. Tsivgoulis G, Katsanos AH, Köhrmann M, Caso V, Lemmens R, Tsioufis $\mathrm{K}$, et al. Embolic strokes of undetermined source: theoretical construct or useful clinical tool? Ther Adv Neurol Disord. (2019) 12:1-12. doi: $10.1177 / 1756286419851381$

39. Kamel H, Longstreth WT, Tirschwell DL, Kronmal RA, Broderick JP, Palesch YY, et al. The AtRial Cardiopathy and Antithrombotic Drugs In prevention After cryptogenic stroke randomized trial: rationale and methods. Int J Stroke. (2019) 14:207-14. doi: 10.1177/17474930187 99981

Conflict of Interest: The authors declare that the research was conducted in the absence of any commercial or financial relationships that could be construed as a potential conflict of interest.

Copyright (c) 2019 Palà, Bustamante, Clúa-Espuny, Acosta, Gonzalez-Loyola, Ballesta-Ors, Gill, Caballero, Pagola, Pedrote, Muñoz and Montaner. This is an open-access article distributed under the terms of the Creative Commons Attribution License (CC BY). The use, distribution or reproduction in other forums is permitted, provided the original author(s) and the copyright owner(s) are credited and that the original publication in this journal is cited, in accordance with accepted academic practice. No use, distribution or reproduction is permitted which does not comply with these terms. 